\title{
A REFINEMENT OF GREEN'S THEOREM ON THE DEFECT GROUP OF A $p$-BLOCK
}

\author{
T. Y. LAM $^{1}$
}

\begin{abstract}
Let $D$ be the defect group of a $p$-block of a finite group $G$. Let $P, Q$ be two $p$-Sylow groups of $G$ containing $D$. Then there exist $x, y, z$ $\in C_{G}(z)$ such that: (i) $z$ is $p$-regular and $D$ is a $p$-Sylow group of $C_{G}(z)$; (ii) $D=Q^{x} \cap P=Q \cap P^{y}$; and (iii) $z=x y$. This refines an earlier theorem of J. A. Green.
\end{abstract}

Let $D$ be the defect group of a $p$-block of a finite group $G$. If $P$ is a $p$-Sylow group of $G$ containing $D$, it has been known for some time that there must exist another $p$-Sylow group $Q$ such that $P \cap Q=D$. This so-called 'Sylow intersection' property was first established by Green [2] using vertex theory, and later also proved by Thompson [4] using Brauer's methods. In [1, p. 241] Alperin indicated that $D$ can even be expressed as a tame intersection of two suitable $p$-Sylow groups of $G$.

In 1968, a much more accurate result was obtained by Green [3]. If $P$ is any given $p$-Sylow group of $G$ containing $D$, Green proved that there must exist $x, y, z \in C_{G}(D)$ such that (i) $z$ is $p$-regular and $D$ is a $p$-Sylow group of $C_{G}(z)$; (ii) $D=P \cap P^{x}=P \cap P^{y}$; (iii) $z=x y$. In particular, if $P$ is chosen such that $N_{P}(D)$ is a $p$-Sylow group of $N_{G}(D)$, then $D=P \cap P^{x}$ in (ii) clearly expresses $D$ as a tame Sylow intersection. On the other hand, the conclusion of (i) recaptures the earlier result of Brauer that $D$ must be a class-defect group of some $p$-regular conjugacy class in $G$.

Green's proof of the above result is a rather elaborate (but extremely successful) application of vertex theory, in the more general framework of $G$ algebras [3]. For $\mathfrak{D}$ a sufficiently large $\mathfrak{p}$-adic ring $(p \mid \mathfrak{p})$, Green views the integral group ring $\Gamma=\Im G$ as a (left) $G \times G$-algebra via the action $\left(g_{1}, g_{2}\right) \cdot \gamma$ $=g_{1} \gamma g_{2}^{-1}\left(g_{i} \in G, \gamma \in \Gamma\right)$. Let $E \in \Gamma$ be the p-adic idempotent associated with the given $p$-block, whose defect group is $D$. Given a $p$-Sylow group $P \supset D$, the following steps are important ingredients in Green's proof:

(1) The vertex of the indecomposable $\supseteq(G \times G)$-module $\Gamma \cdot E$ is $\Delta(D)$ $=\{(d, d): d \in D\}$.

(2) When viewed as $\mathfrak{D}(P \times P)$-module by restriction, $\Gamma \cdot E$ has at least one indecomposable constituent with vertex $=\Delta(D) \subset P \times P$.

(3) Let $\cup_{w \in W} P w P$ be the $(P, P)$-double coset decomposition of $G$, and let $[P w P]$ denote the $\mathcal{S}(P \times P)$-submodule of $\Gamma$, with $P w P$ as $\mathcal{D}$-basis. Then,

Received by the editors November 22, 1974.

AMS (MOS) subject classifications (1970). Primary 20C20, 20D20.

Key words and phrases. $p$-Sylow groups, defect groups, indecomposable modules, vertices, tame Sylow intersections.

${ }^{1}$ Supported in part by the National Science Foundation. 
$\Gamma=\bigoplus_{w \in W}[P w P]$ is a Krull-Schmidt decomposition of $\Gamma$ in the category of $\checkmark(P \times P)$-modules. The vertex of $[P w P]$ is $P(w)=\left\{\left(p_{1}, p_{2}\right) \in P \times P: p_{1} w p_{2}^{-1}\right.$ $=w$. Thus, by (2), at least one such $P(w)$ must be conjugate in $P \times P$ to $\Delta(D)$. We may assume $\Delta(D)=P\left(w_{1}\right)$, and let $\left\{w_{1}, \ldots, w_{c}\right\}$ be a maximal subset of $W$ such that $P\left(w_{j}\right)(1 \leqslant j \leqslant c)$ are mutually nonconjugate in $P \times P$. Since $P\left(p_{1}^{-1} w p_{2}\right)=P(w)^{\left(p_{1}, p_{2}\right)}$, we may assume $W$ to have been so chosen that each $P(w)(w \in W)$ is actually equal (not just conjugate) to some (unique) $P\left(w_{j}\right)$.

(4) The endomorphism algebra $\operatorname{End}_{P \times P} \Gamma$, modulo its radical, can be explicitly determined, in the form $\prod_{j=1}^{c} \mathbf{M}_{m_{j}}(\Im / \mathfrak{p})$, a product of matrix algebras over the modular field $\subseteq / \mathfrak{p}$. To be precise, the matrices in the $j$ th factor have rows and columns suffixed by $W_{j}=\left\{w \in W: P(w)=P\left(w_{j}\right)\right\}$, and so $m_{j}=\left|W_{j}\right|$.

(5) By left multiplication, the center of $\Gamma$ acts as $G \times G$ - (hence $P \times P$-) endomorphisms on $\Gamma$. Using the projections $\operatorname{End}_{P \times P} \Gamma \rightarrow \mathbf{M}_{m_{j}}(\Im / \mathfrak{p})$ in (4), one obtains a family of 'representations' $\Psi_{j}$ : Center $\Gamma \rightarrow \mathbf{M}_{m_{j}}(\mathfrak{D} / \mathfrak{p})$. If $\mathscr{\Omega}$ is a typical conjugacy class in $G$, and $K$ is its class sum in $\Gamma$, then $\Psi_{j}(K)$ will be a matrix whose $(u, v)$-entry $\left(u, v \in W_{j}\right)$ is the cardinality of the following set $($ taken $\bmod p)$ :

$$
F_{u, v}^{j}(\Re ; P)=\left\{x \in P u P: P(x)=P\left(w_{j}\right) \text { and } x v^{-1} \in \Re\right\} .
$$

(6) On the other hand, $\Psi_{j}$ sends the given $\mathfrak{p}$-adic idempotent $E$ to a matrix $\Psi_{j}(E)$ whose rank happens to be the number of indecomposable constituents of $(\Gamma \cdot E)_{P \times P}$ with vertex conjugate in $P \times P$ to $P\left(w_{j}\right)$.

The proofs of the above facts vary in degree of difficulty; taken together, they provide an extremely useful tool with which to study the defect group $D$.

In the following, we shall show how the above information can be further strengthened. Namely, instead of working with one $p$-Sylow group $P \supset D$, one could work simultaneously with two given $p$-Sylow groups $P \supset D, Q \supset D$. By recasting Green's original methods, we shall show that the following refinement of Green's theorem holds:

THEOREM . Let $D$ be the defect group of a p-block of the finite group $G$. Let $P, Q$ be two $p$-Sylow groups of $G$ containing $D$. Then there exist $x, y, z \in C_{G}(D)$ such that: (i) $z$ is p-regular and $D$ is a p-Sylow group of $C_{G}(z)$; (ii) $D=Q^{x} \cap P$ $=Q \cap P^{y}$, and (iii) $z=x y$.

To establish this, it is essential to extend the facts (1) through (6) above to cope with the situation of two p-Sylow groups. The point is that, because of the way the $G \times G$ action on $\Gamma$ is defined, it is just as easy (and obviously more effective) to study the restriction of $\Gamma$ and $\Gamma \cdot E$ to the subgroup $Q \times P$ $\subset G \times G$. Fact (1) remains, of course, unchanged. For (2), we now have $\Delta(D) \subset Q \times P$, and, by Green's theory, the restriction $(\Gamma \cdot E)_{Q \times P}$ still has at least one indecomposable constituent with vertex $=\Delta(D)$. For (3), we must now let $W$ be a full set of $(Q, P)$-double coset representatives in $G$ (instead of $(P, P)$-coset representatives). Notice that $Q \times P$ is a $p$-group, and $[Q w P]$ (with the obvious definition) is a transitive permitation module over $Q \times P$. By an 
earlier theorem of Green [2, Lemma 2.3a], $[Q w P]$ is still $\unlhd(Q \times P)$-indecomposable. Hence, $\Gamma=\bigoplus_{w \in W}[Q w P]$ remains a Krull-Schmidt decomposition in the category of $\subseteq(Q \times P)$-modules. For $x \in G$, let $J(x)=\{(a, b) \in Q \times P$ : $\left.a x b^{-1}=x\right\}$, the subgroup of $Q \times P$ fixing $x$. (Green's $P(x, y)$ in [3, p.145] should be correspondingly changed into a more general $J(x, y)$, but we still have $J(x, y)=J(x) \cap J(y), \ldots$, etc.) Letting $\left\{w_{1}, \ldots, w_{c}\right\}$ be a maximal subset of $W$ such that $J\left(w_{j}\right)$ are mutually nonconjugate in $Q \times P$, we may assume, by what we said so far, that $\Delta(D)=J\left(w_{1}\right)$. Again, $W$ may be 'normalized' so that each $J(w)(w \in W)$ equals some (unique) $J\left(w_{j}\right)$.

The endomorphism algebra $\mathrm{End}_{Q \times P} \Gamma$, modulo its radical, can be calculated along essentially the same lines as in [3], with minor notational changes. The quotient breaks up into a product $\prod_{j=1}^{c} \mathbf{M}_{m_{j}}(\mathfrak{D} / \mathfrak{p})$, where the matrices in the $j$ th factor are suffixed by the set $W_{j}=\left\{w \in W: J(w)=J\left(w_{j}\right) \subset Q \times P\right\}$.

By a procedure similar to that used in (5), one obtains again the representations $\Psi_{j}$. For the class sum $K$ of any conjugacy class $\Re$, one can show that $\Psi_{j}(K)$ is evaluated as before, after replacing the old $F_{u, v}^{j}(\Re ; P)$ by new sets:

$$
F_{u, v}^{j}(\Re ; Q, P)=\left\{x \in Q u P: J(x)=J\left(w_{j}\right) \text { and } x v^{-1} \in \Re\right\} \quad\left(u, v \in W_{j}\right) .
$$

Finally, the new version of (6), proved by rather routine changes of Green's methods, now states that $\Psi_{j}(E)$ has rank equal to the number of indecomposable constituents of $(\Gamma \cdot E)_{Q \times P}$ with vertex conjugate in $Q \times P$ to $J\left(w_{j}\right)$.

Having said all the above, the proof of the Theorem now proceeds as follows (cf. [3, p.149]). Let $\Re_{i} \quad(1 \leqslant i \leqslant n)$ be the conjugacy classes of $G$, with sums $K_{i}$, and let $E=\sum_{i}^{n} a_{i} K_{i}$. Since $\Delta(D)=J\left(w_{1}\right)$, the new versions of (2) and (6) imply that $\Psi_{1}(E)$ has rank $\geqslant 1$. We have $0 \neq \Psi_{1}(E)=\sum_{i} \bar{a}_{i} \Psi_{1}\left(K_{i}\right)$ ('bar' denotes mod $\mathfrak{p}$ ), so there must exist a class $\Re_{h}$ for which $\bar{a}_{h} \neq 0$ and $\Psi_{1}\left(K_{h}\right) \neq 0$. The former implies (as is well known) that $\Omega_{h}$ is $p$-regular, and the latter implies, thanks to the new version of $(5)$, that the sets $F_{u, v}^{1}\left(\Omega_{h} ; Q, P\right)$ cannot be all empty for $u, v \in W_{1}$. Choose $u, v \in W_{1}$ for which there exists $x \in F_{u, v}^{1}\left(\Re_{h} ; Q, P\right)$. We have then $J(x)=J(v)=J\left(w_{1}\right)=\Delta(D)$. But $J(x)$ $=\left\{(a, b) \in Q \times P: a^{x}=b\right\}$. Thus, $J(x)=\Delta(D)$ implies that $D=Q^{x} \cap P$ and $x \in C_{G}(D)$. Similarly, we obtain $D=Q^{v} \cap P$ and $v \in C_{G}(D)$. Setting $y=v^{-1} \in C_{G}(D)$, we have $D=Q \cap P^{y}$. The element $z=x y=x v^{-1}$ $\in \Re_{h}$ is, therefore, $p$-regular, and its class-defect group contains $D$. But then $D$ must actually equal the class-defect group of $\Re_{h}$. Q.E.D.

Finally, from the Theorem just proved, we may record the following generalization of the 'tame-Sylow-intersection' property:

COROLlary . Let $D$ be the defect group of a p-block of the finite group $G$, and let $P$ be any $p$-Sylow group of $G$ containing $D$. Then there exists a $p$-Sylow group $S$ of $G$ such that $D=S \cap P$, and $N_{S}(D)$ is a p-Sylow subgroup of $N_{G}(D)$.

Proof. Choose a $p$-Sylow group $Q \supset D$ such that $N_{Q}(D)$ is a $p$-Sylow group of $N_{G}(D)$. There exists, by the Theorem, $x \in C_{G}(D)$ such that $D=Q^{x} \cap P$. The new $p$-Sylow group $S=Q^{x}$ clearly has the desired properties. Q.E.D. 


\section{REFERENCES}

1. J. L. Alperin, Sylow intersections and fusion, J. Algebra 6 (1967), 222-241. MR 35 \#6748.

2. J. A. Green, Blocks of modular representations, Math. Z. 79 (1962), 100-115. MR 25 \# 5114.

3. - Some remarks on defect groups, Math. Z. 107 (1968), 133-150. MR 38 \#222.

4. J. G. Thompson, Defect groups are Sylow intersections, Math. Z. 100 (1967), 146. MR 35 \#4296.

Department of Mathematics, University of California, Berkeley, California 94720 